\title{
Expression of p63 in the Mouse Ovary
}

\author{
Nobuaki NAKAMUTA ${ }^{1)}$ and Shigeru KOBAYASHI ${ }^{1)}$ \\ 1)Department of Biosciences, Kyushu Dental College, Kitakyushu 803-8580, Japan
}

\begin{abstract}
Transcription factor p63, a member of the tumor suppressor p53 family, plays an important role in epithelial development, and its expression is found in many epithelial tissues. Our previous reports have shown that p63 is expressed in primordial and testicular germ cells. In this study, we investigated the expression and distribution of p63 isoforms (TAp63 $\alpha$, TAp63 $\beta$, TAp63 $\gamma, \Delta \mathrm{Np} 63 \alpha$, $\Delta \mathrm{Np} 63 \beta$ and $\Delta \mathrm{Np} 63 \gamma$ ) in the mouse ovary. Reverse transcription-polymerase chain reaction experiments with primers specific for different p63 isoforms demonstrated that transcripts encoding the transactivation domain and $\alpha$ isoforms were preferentially expressed in the ovary. Immunolocalization of p63 proteins, presumably that of full-length p63 $\alpha$ isoform, was observed in the oocytes of primordial, primary and two-layered secondary follicles. However, positive staining was not detected in the oocytes of multi-layered secondary follicles and antral follicles as they continued to develop. This data suggests that p63 may be implicated in the meiosis and cell cycle control of germ cells in the mouse ovary.
\end{abstract}

Key words: Apoptosis, Cell cycle, Mouse, Ovary, Transcription factor p63

(J. Reprod. Dev. 53: 691-697, 2007)

$T^{\mathrm{n}}$ ranscription factor $\mathrm{p} 63$, a member of the p53 family, has significant sequence similarity with tumor suppressor p53 [1-5]. Like $p 53$, the $p 63$ gene encodes an amino-terminal transactivation domain, central DNA binding domain, and carboxyterminal oligomerization domain. Unlike $p 53$, however, the $p 63$ gene has two promoters and generates two types of transcripts with (TAp63) or without $(\Delta \mathrm{Np} 63)$ a transactivation domain. While TAp63 can induce cell cycle arrest and apoptosis, $\Delta \mathrm{Np} 63$ has dominant-negative effects in inhibiting the transcription activating function of TAp63 and p53. Each mRNA gives rise to three different carboxy termini, designated $\alpha, \beta$ and $\gamma$, by alternative splicing. Therefore, six isoforms of p63 may exist. Both TAp63 $\alpha$ and $\Delta \mathrm{Np} 63 \alpha$ contain an additional region called the sterile alpha motif

Accepted for publication: January 16, 2007

Published online: February 23, 2007

Correspondence: N. Nakamuta (e-mail: nakamuta@kyudent.ac.jp) domain. This domain is located near the carboxy terminus of the $\alpha$ isoform of p63 and is a proteinprotein interaction domain involved in developmental processes [6].

Many studies have shown that p63 plays an important role in ectodermal development [7]. In the early period of embryonic development, p63 expression has been demonstrated in the surface ectoderm of pharyngeal arches and apical ectodermal ridges of the limb buds [8,9]. Their obvious importance in the developmental process is also suggested by the p63-knockout mouse, which is characterized by striking defects in its limbs and craniofacial development $[8,9]$. In humans, $p 63$ gene mutations lead to congenital defects, such as the ankyloblepharon-ectodermal dysplasia-clefting (AEC) and ectrodactyly ectodermal dysplasia and facial clefting (EEC) syndromes $[10,11]$. However, expression of $p 63$ mRNAs is not restricted to the skin. As has been depicted by Northern blot analysis of human 
tissues, $p 63$ mRNAs have also been detected in a variety of tissues, including skeletal muscle and the placenta, heart, thymus, trachea and uterus [4, 12]. Our previous studies have revealed germ cell expression of p63 in the mouse testis. Reverse transcription-polymerase chain reaction (RT-PCR) analyses of testicular RNAs have shown that TAp63 is constitutively expressed in the mouse testis during postnatal development, while $4 N p 63$ is expressed at several specific stages of development [13]. Testicular expression of $\triangle N p 63 \mathrm{mRNA}$ is restricted to the newborn ( $0-1$ week of age) and immature (3-4 week of age) periods. This coincides with the initiation of spermatogenic cell proliferation and the peak of germ cell apoptosis, respectively. Furthermore, p63 proteins have been demonstrated in spermatocytes and spermatids up to adulthood using immunohistochemistry, with first appearance 2 weeks (spermatocytes) and 3 weeks (spermatids) after birth [13]. These expression patterns of p63 in the male gonads suggest that p63 may participate in regulation of meiosis, cell cycle arrest, and apoptosis. In contrast to the testis, however, expression of p63 in ovarian tissues has not been explored in detail. The process of gametogenesis is strikingly different between males and females. For instance, haploid germ cells are repeatedly produced from stem cell spermatogonia by mitotic and meiotic cell divisions in the adult testis. In contrast, germ cells in the ovaries enter meiosis before birth, and arrest at the prophase of the first meiotic division, which do not complete until a few hours before ovulation. Therefore, RT-PCR and immunohistochemical analyses of p63 expression were performed in this study to elucidate the temporal and spatial patterns of p63 expression in the mouse ovary.

\section{Materials and Methods}

Female ICR mice were purchased from Clea Japan (Tokyo, Japan) and kept at the Animal Research Center of Kyushu Dental College until use. The animals were sacrificed by cervical dislocation under diethyl ether anesthesia. Brains, thymuses, lungs, hearts, livers, spleens, kidneys, and ovaries were immediately dissected and either processed for RNA extraction or immersed in Bouin's fixative. All procedures were approved by the intramural committee for the use of laboratory animals, and the mice were treated in accordance with the Guidelines for the Care and Use of Laboratory Animals at Kyushu Dental College.

Total RNAs were extracted from adult mouse tissues using Isogen (Nippon Gene, Tokyo, Japan). Reverse transcription of $1 \mu \mathrm{g}$ RNA was performed using Oligo-dT Primers and SuperScript II Reverse Transcriptase (Invitrogen, Carlsbad, CA, USA). The primers and PCR conditions used for TAp63, $\Delta N p 63, p 63 \alpha, p 63 \beta, p 63 \gamma$ and glyceraldehyde-3phosphate dehydrogenase (GAPDH; used as internal control) were described previously [13, 14]. Briefly, the primer sequences used for amplification of TAp63 were forward 5'-CGAAAATGGTGCAACAAACA-3' (Genbank accession number AF075436, 243-262) and reverse 5'-GATGGAGAGAGGGCATCAAA-3' (466-485). The primers for $\triangle N p 63$ were forward 5'-GGAAAACAATGCCCAGACTC-3' (AF075439, 12-31) and reverse 5'-GATGGAGAGAGGGCATCAAA-3' (184-203). The primers for p63 $\alpha$ were forward 5'-AGTACCTCCCTCAGCACACG-3' (AF075439, 992-1011) and reverse 5'CCTGGGTCGTGAAATAGTCC-3' (1404-1423). The primers for $p 63 \beta$ were forward $5^{\prime}$ AGTACCTCCCTCAGCACACG-3' (AF075438, 9921011) and reverse 5'-GCCAAATCCTGACAATGCTG-3' (1359-1378). The primers for $p 63 \gamma$ were forward 5'-AGTACCTCCCTCAGCACACG-3' (AF075437, 980-999) and reverse 5'ATGGGTACACGGAGTGGTTT-3' (1149-1168). The primers for GAPDH were forward $5^{\prime}$ ATGGTGAAGGTCGGTGTGAAC-3' (MMGAPDH, 47-67) and reverse 5'-GGCTTGACTGTGCCGTTGAAT-3' (205-225). PCR consisted of an initial denaturing step of $5 \mathrm{~min}$ at $95 \mathrm{C} ; 35$ cycles (or 24 cycles for GAPDH) of $30 \mathrm{sec}$ at $95 \mathrm{C}, 30 \mathrm{sec}$ at $55 \mathrm{C}$ (or $57 \mathrm{C}$ for $T A$ and $\triangle N p 63$ ), $1 \mathrm{~min}$ at $72 \mathrm{C}$; and a 3 min extension step at $72 \mathrm{C}$. The cDNA samples were subjected to PCR using specific primers designed to amplify a 241 bp product for TAp63, $190 \mathrm{bp}$ product for $\triangle N p 63,432 \mathrm{bp}$ for $p 63 \alpha, 387 \mathrm{bp}$ for $p 63 \beta, 189 \mathrm{bp}$ for $p 63 \gamma$ and $179 \mathrm{bp}$ for GAPDH. The amplified products were resolved in 1.5\% agarose gels, stained with ethidium bromide, and photographed under ultraviolet transillumination light using a TFX 20M UV Transilluminator (Vilber Lourmat, Marne-la-Vallee, France).

Primary antibodies were purchased from Santa Cruz Biotechnology (Santa Cruz, CA, USA). Mouse monoclonal antibody pan-p63 (4A4), which reacts with all p63 isoforms, is raised against amino acids 
1-205 mapping at the amino terminus of human $\Delta$ Np63. TAp63 (D-20), which reacts with TAp63 $\alpha$, $\beta$ and $\gamma$, is a goat polyclonal antibody raised against a peptide mapping near the amino terminus of mouse TAp63. $\Delta \mathrm{Np} 63(\mathrm{~N}-16)$, which reacts with $\Delta \mathrm{Np} 63 \alpha, \beta$ and $\gamma$, is a goat polyclonal antibody raised against a peptide mapping near the amino terminus of mouse $\Delta \mathrm{Np} 63 \alpha$. p63 $\alpha$ (H-129), which reacts with $\mathrm{TAp} 63 \alpha$ and $\Delta \mathrm{Np} 63 \alpha$, is a rabbit polyclonal antibody raised against a recombinant protein corresponding to amino acids 513-641 mapping at the carboxy terminus of human $p 63 \alpha$. p63 $\gamma(\mathrm{C}-18)$, which reacts with TAp63 $\gamma$ and $\Delta \mathrm{Np} 63 \gamma$, is a goat polyclonal antibody raised against a peptide mapping at the carboxy terminus of human p $63 \gamma$. Immunohistochemical analysis was performed as described previously [15]. Briefly, paraffin sections were mounted on silane-coated glass slides. Unmasking of epitopes was achieved by heating in $0.01 \mathrm{M}$ sodium citrate $(\mathrm{pH}$ 6.0). The endogenous peroxidase was quenched with $0.3 \%$ $\mathrm{H}_{2} \mathrm{O}_{2}$ in methanol. The sections were treated with non immune serum to avoid nonspecific bindings, incubated with primary antibodies overnight, and then incubated with the horseradish peroxidase (HRP)-conjugated secondary antibodies for $30 \mathrm{~min}$. The HRP-reaction was developed using diaminobenzidine tetrahydrochloride (Dojindo, Kumamoto, Japan). Negative controls included primary antibodies preincubated with a ten-fold excess of the respective blocking peptides and incubation with phosphate buffered saline instead of the primary antibodies.

\section{Results}

We examined $p 63$ expression in multiple mouse tissues by RT-PCR. As an internal control for RNA extraction and CDNA synthesis, PCR amplification for a housekeeping gene, GAPDH, was also performed on all cDNA samples. RT-PCR showed that $p 63$ isoforms were differentially expressed in various tissues of the adult mouse (Fig. 1). The TA isoform was expressed in the brain, thymus, kidney and ovary. In contrast, the $\Delta N$ isoforms were expressed in the thymus, lung and kidney. In addition, the $\alpha$ isoform was expressed in the brain, thymus, lung, heart, kidney and ovary, while the $\beta$ and $\gamma$ isoforms were not detected in any of the samples examined. Therefore, the transcripts

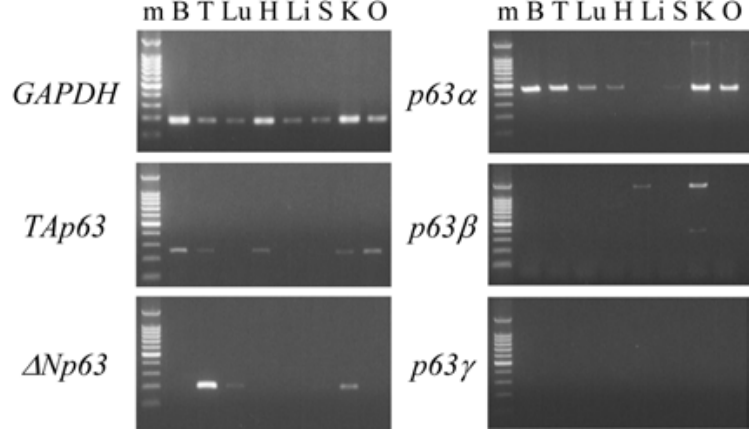

Fig. 1. Different $p 63$ isoforms are expressed in different tissues. Total RNAs were prepared from mouse tissues including the brain (B), thymus (T), lung $(\mathrm{Lu})$, heart $(\mathrm{H})$, liver $(\mathrm{Li})$, spleen $(\mathrm{S})$, kidney $(\mathrm{K})$ and ovary $(\mathrm{O})$. TAp63 is expressed in the brain, thymus, kidney and ovary, whereas $\triangle N p 63$ is expressed in the thymus, lung and kidney. The $\alpha$ isoform of p63 is expressed in several tissues, including the brain, thymus, lung, heart, kidney and ovary; the $\beta$ and $\gamma$ isoforms were not detected in any of the tissues tested. Note that the bands in the lanes for the liver and kidney in the $p 63 \beta$ panel are nonspecific. Control RT-PCR for GAPDH is also shown. The bands for the $100 \mathrm{bp}$ ladder (m) are also indicated.

encoding for the TA and $\alpha$ isoforms were predominant in the ovary.

Subsequently, immunohistochemistry using a panel of antibodies against p63 (pan-p63, TAp63, $\Delta \mathrm{Np} 63, \mathrm{p} 63 \alpha$ and $\mathrm{p} 63 \gamma$ ) was performed to examine the protein distribution of the p63 isoforms in the ovary. Small oocytes of the primordial follicles were surrounded by a layer of flattened pregranulosa cells (Fig. 2A1). Larger oocytes of the primary follicles were surrounded by a single layer of cuboidal granulosa cells (Fig. 2A2). Preantral follicles were classified as two-layered secondary (Fig. 2A3) or multi-layered secondary follicles (Fig. 2A4) depending on the numbers of granulosa cell layers. These follicles were surrounded by theca cells. Antral follicles consisted of a large oocyte surrounded by cumulus cells, a fluid-filled antrum, granulosa cells and theca cells (Fig. 2A5).

Immunohistochemical analysis using pan-p63 antibody showed that the nuclei of the oocytes within primordial and primary follicles were positive for p63 (Fig. 2B1 and 2). Positive staining for pan-p63 was also observed in the small, twolayered secondary follicles (Fig. 2B3). However, p63 expression was not recognized within multi- 


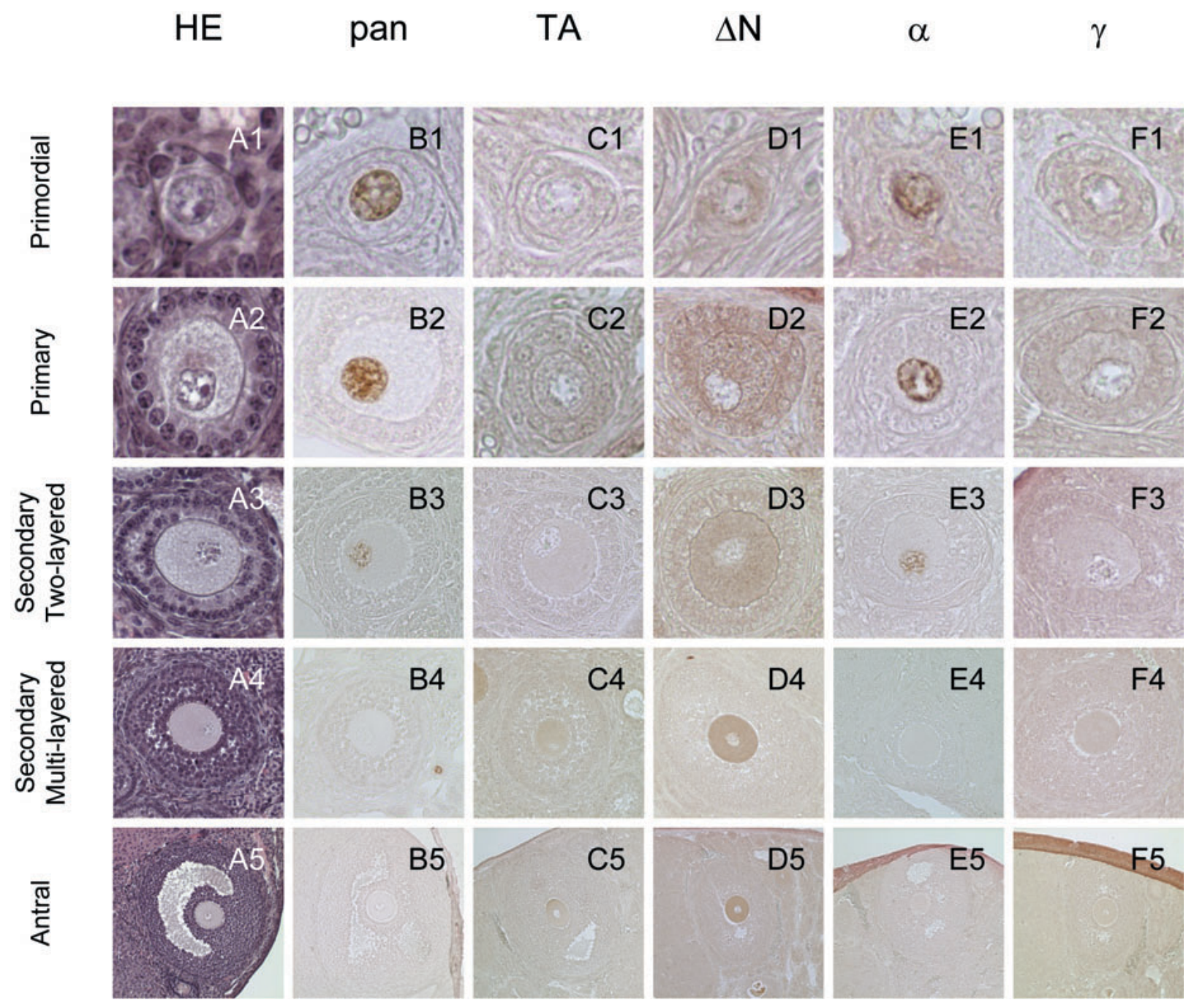

Fig. 2. Immunolocalization of $\mathrm{p} 63$ proteins in adult mouse ovaries. The oocytes of the primordial, primary and two-layered secondary follicles are intensely stained for pan-p63 antibody (B1-3). However, no significant p63 expression is recognizable within the multi-layered secondary follicle (B4) and antral follicle (B5). Positive staining can be observed in the primordial, primary and two-layered follicles with p $63 \alpha$. No staining can be seen in the ovary using immunohistochemistry with the TA (C1-5), $\triangle \mathrm{N}(\mathrm{D} 1-5)$ or p63 $\gamma(\mathrm{F} 1-5)$ antibodies. Original magnifications: $\times 80(\mathrm{~A} 1-\mathrm{F} 1$ and A2-F2), $\times 40(\mathrm{~A} 3-\mathrm{F} 3), \times 20(\mathrm{~A} 4-\mathrm{F} 4)$ and $\times 10(\mathrm{~A} 5-\mathrm{F} 5)$.

layered secondly follicles (Fig. 2B4). Atretic follicles with apoptotic granulosa cells were also negative for p63 (not shown). No significant p63positive reactions were recognized in antral follicles in which a fluid-filled antral space had formed (Fig. 2B5). No positive reactions were seen in corpora lutea as well (not shown). Immunostaining for pan-p63 was restricted to germ cells in the mouse ovary throughout the entire period of examination.

Although the mRNA for TAp63 was detected by
RT-PCR, immunohistochemistry could not detect TAp63 protein in the ovary (Fig. 2C1-5). Indeed, some of the oocytes within secondary follicles showed faint TAp63 immunoreactivity in their cytoplasm (not shown). However, this proved to be false-positive staining since identical results were obtained when the primary antibody was preadsorbed with its immunizing peptide.

No staining was apparent in the ovary with anti$\Delta$ Np63 antibody (Fig. 2D1-5). However, faint staining for $\Delta \mathrm{Np} 63$ was present in the cytoplasm of 
the oocytes (Fig. 2D4 and 5). Although this immunoreactivity became weak or was lost in absorption test using its immunizing peptide, we considered this $\Delta \mathrm{N}$ staining to be negligible since no cytoplasmic staining was recognized in oocytes using an antibody that can detect all p63 isoforms (Fig. 2B4 and 5).

The oocytes of primordial, primary, and twolayered secondary follicles showed intense staining for p63 $\alpha$ (Fig. 2E1-3). However, they were negative for $\mathrm{p} 63 \alpha$ in multi-layered secondary follicles and antral follicles (Fig. 2E4 and 5).

In agreement with the results of our mRNA analysis, p63 $\gamma$ immunoreactivity was not detected in the ovary (Fig. 2F1-5).

\section{Discussion}

We obtained results indicating the presence of mRNAs encoding the TA and $\alpha$ variants in the mouse ovary (Fig. 1). We prepared total RNA from various mouse tissues and performed RT-PCR specific for different p63 isoforms. Only the TA isoform was expressed in the brain, heart and ovary, whereas only the $\triangle N p 63$ isoform was present in the lung. Both the TA and $\triangle N$ isoforms were present in the thymus and kidney. This expression pattern for $p 63$ mRNAs essentially confirms that in a previous report [5]. Our RT-PCR analysis also indicated that $p 63 \alpha \mathrm{mRNA}$ is widely expressed in adult mouse tissues. This analysis revealed the presence of transcripts encoding $p 63 \alpha$ in the brain, thymus, lung, heart, kidney and ovary. No $p 63 \alpha$ mRNA expression was detected in the liver or spleen. In addition, the $\beta$ and $\gamma$ isoforms were not amplified in any of the samples examined. Collectively, the present RT-PCR data indicates that the TA and $\alpha$ isoforms of p63 are preferentially expressed in the mouse ovary. Although we could not detect $p 63 \gamma$ mRNA in ovary, Kurita et al. [16] detected mRNA for $p 63 \gamma$ in the mouse ovary by RTPCR. This might be due to a difference in the sensitivity of the detection method employed, and thus, mRNA for the $\gamma$ isoform may also be present at a low level in the ovary. However, immunohistochemical analysis showed no immunoreactivity for anti-p63 $\gamma$ antibody in the mouse ovary.

Consistent with previous reports [16, 17], immunohistochemical staining with anti-p63 antibodies demonstrated the expression of p63 proteins in the oocytes (Fig. 2). Identical results were obtained when immunohistochemistry was performed using two different antibodies. One antibody was reactive to all six isoforms and the other was specific to the carboxy terminus of $\alpha$ isoforms. As shown in Fig. 2B1-3 and E1-3, the oocytes in primordial, primary, and secondary follicles stained positively for pan-p63 and p63 $\alpha$. Our immunohistochemical examination did not detect TAp63 in the mouse ovary (Fig. 2C1-5); however, Suh et al. [17] reported that mouse oocyte nuclei stained positively for p63 in an immunohistochemical analysis using an antibody specific to the transactivation domain of TAp63. The anti-TAp63 antibody used in their study was generated by immunization of the mouse with a fusion protein of glutathione $S$-transferase and the first 69 amino acid residues of TAp63. On the other hand, a distinct antibody was used here to detect TAp63 proteins. We used a goat polyclonal antibody TAp63, which is specific to the amino terminus of TAp63. Therefore, the discrepancy between the results may be attributable to the difference in the two antibodies and/or detection methods. As the $\Delta \mathrm{N}$ and $\gamma$ antibodies did not show any significant staining in the ovary (Fig. 2D1-5 and F1-5), our immunohistochemical and RT-PCR findings were in agreement.

Previous studies have shown expression of $p 63$ mRNAs in the mouse testis and nuclear localization of p63 proteins in testicular germ cells $[13,15]$. Additionally, p63 has been detected in the nuclei of mouse primordial germ cells by immunohistochemistry [14]. In conjunction with the present data, these results imply that p63 is profoundly involved in the regulation of germ cell development. The p63 proteins have been shown to be expressed in the basal cells of many epithelial tissues $[5,18]$ and are thought to play essential roles in maintaining epidermal stem cell populations [7]. However, the roles of p63 in germ cells are not fully understood. Particularly, their ability to make sperms or eggs has not been addressed because p63-null mice die shortly after birth $[8,9]$.

During the course of this study, two papers were published showing the presence of normal ovaries in $p 63-/-$ mouse fetuses [16, 17]. Furthermore, follicles developed when $p 63-/-$ ovaries were grafted into host mice, suggesting that p63 is not necessary for the development of female germ cells. 
However, it is important to note that p63 and other members of the p53 family share high homology with each other and have overlapping functions. Therefore, in $p 63$-deficient mice, $p 53$ or $p 73$ may have been used to accomplish the functions normally provided by $\mathrm{p} 63$.

Considering the expression patterns we described, p63 might have several functions in the ovaries. One possibility is that p63 may control the meiosis as indicated by their germ cell-specific expression in the ovary. This hypothesis is supported by the observation that p63 proteins are present in pre- and postmeiotic germ cells in the mouse testis $[13,19]$. In addition, p63 may be involved in the cell cycle control of oocytes since the positive reaction for p63 was lost in the oocytes as follicles developed. In support of this possibility, it has been demonstrated that p63 can induce cell cycle arrest through activation of the p53 target genes in vitro [20]. In the testes, in which the cell cycle of germ cells stops during the fetal period, the level of p63 expression declines and diminishes prior to resumption of germ cell proliferation after birth $[13,15]$. Thus, despite the significant difference between male and female gametogenesis, p63 protein is expressed in the quiescent stage of germ cell development and diminishes before the germ cells resume cell cycle progression, suggesting their possible role in regulation of germ cell development. Therefore, p63 may be involved in cell cycle control of oocytes being arrested in the first meiotic prophase until the follicles begin to grow.

In conclusion, the present RT-PCR and immunohistochemical analyses for the p63 isoforms in the mouse ovary suggest expression and nuclear localization of TAp63 $\alpha$ in oocytes at specific stages of follicle development. This data also suggests that p63 may be implicated in the meiosis and cell cycle control of female germ cells. Further detailed studies are needed to elucidate the ability of $p 63-/-$ female germ cells to develop and to determine whether the oocytes are fertile. It is also important to identify the p63 target genes and the partner of protein-protein interaction within germ cells and to clarify the signaling cascade that regulates germ cell expression of p63.

\section{References}

1. Schmale $\mathbf{H}$, Bamberger $\mathbf{C}$. A novel protein with strong homology to the tumor suppressor p53. Oncogene 1997; 15: 1363-1367.

2. Senoo M, Seki N, Ohira M, Sugano S, Watanabe M, Inuzuka S, Okamoto T. A second p53-related protein, p73L, with high homology to p73. Biochem Biophys Res Commun 1998; 248: 603-607.

3. Trink B, Okami K, Wu L, Sriuranpong V, Jen J, Sidransky D. A new human p53 homologue. Nat Med 1998; 4: 747-748.

4. Osada M, Ohba M, Kawahara C, Ishioka C, Kanamaru R, Katoh I, Ikawa Y, Nimura Y, Nakagawara A, Obinata M, Ikawa S. Cloning and functional analysis of human p51, which structurally and functionally resembles p53. Nat Med 1998; 4: 839-843.

5. Yang A, Kaghad M, Wang Y, Gillett E, Fleming MD, Dotsch V, Andrews NC, Caput D, McKeon F. p63, a p53 homolog at 3q27-29, encodes multiple products with transactivating, death-inducing, and dominant-negative activities. Mol Cell 1998; 2: 305316.

6. Thanos CD, Bowie JU. p53 Family members p63 and p73 are SAM domain-containing proteins. Protein Sci 1999; 8: 1708-1710.
7. Koster MI, Roop DR. The role of p63 in development and differentiation of the epidermis. $J$ Dermatol Sci 2004; 34: 3-9.

8. Mills AA, Zheng B, Wang XJ, Vogel H, Roop DR, Bradley A. p63 is a p53 homologue required for limb and epidermal morphogenesis. Nature 1999; 398: 708-713.

9. Yang A, Schweitzer R, Sun D, Kaghad M, Walker N, Bronson RT, Tabin C, Sharpe A, Caput D, Crum C, McKeon F. p63 is essential for regenerative proliferation in limb, craniofacial and epithelial development. Nature 1999; 398: 714-718.

10. McGrath JA, Duijf PH, Doetsch V, Irvine AD, de Waal R, Vanmolkot KR, Wessagowit V, Kelly A, Atherton DJ, Griffiths WA, Orlow SJ, van Haeringen A, Ausems MG, Yang A, McKeon F, Bamshad MA, Brunner HG, Hamel BC, van Bokhoven H. Hay-Wells syndrome is caused by heterozygous missense mutations in the SAM domain of p63. Hum Mol Genet 2001; 10: 221-229.

11. Celli J, Duijf P, Hamel BC, Bamshad M, Kramer B, Smits AP, Newbury-Ecob R, Hennekam RC, Van Buggenhout $G$, van Haeringen $A$, Woods $C G$, van Essen AJ, de Waal R, Vriend G, Haber DA, Yang A, McKeon F, Brunner HG, van Bokhoven $H$. 
Heterozygous germline mutations in the p53 homolog p63 are the cause of EEC syndrome. Cell 1999; 99: 143-153.

12. Dellavalle RP, Egbert TB, Marchbank A, Su LJ, Lee LA, Walsh P. CUSP/p63 expression in rat and human tissues. J Dermatol Sci 2001; 27: 82-87.

13. Nakamuta $\mathbf{N}$, Kobayashi $\mathbf{S}$. Developmental expression of p63 in the mouse testis. J Vet Med Sci 2004; 66: 681-687.

14. Nakamuta N, Kobayashi S. Expression of p63 in the mouse primordial germ cells. J Vet Med Sci 2004; 66: 1365-1370.

15. Nakamuta N, Kobayashi S. Expression of p63 in the testis of mouse embryos. J Vet Med Sci 2003; 65: 853856.

16. Kurita T, Chunha GR, Robboy SJ, Mills AA, Medina RT. Differential expression of p63 isoforms in female reproductive organs. Mech Dev 2005; 122: 1043-1055.

17. Suh EK, Yang A, Kettenbach A, Bamberger C, Michaelis AH, Zhu Z, Elvin JA, Bronson RT, Crum CP, McKeon F. p63 protects the female germ line during meiotic arrest. Nature 2006; 444: 624-628.

18. Parsa R, Yang A, McKeon F, Green H. Association of p63 with proliferative potential in normal and neoplastic human keratinocytes. J Invest Dermatol 1999; 113: 1099-1105.

19. Hamer G, Gademan IS, Kal HB, de Rooij DG. Role for $\mathrm{c}-\mathrm{Abl}$ and $\mathrm{p} 73$ in the radiation response of male germ cells. Oncogene 2001; 20: 4298-4304.

20. Dohn $\mathbf{M}$, Zhang $\mathbf{S}$, Chen $\mathbf{X}$. p63alpha and DeltaNp63alpha can induce cell cycle arrest and apoptosis and differentially regulate p53 target genes. Oncogene 2001; 20: 3193-3205. 